\title{
Real Space Imaging and Molecular Packing of Dendronized Polymer-Lipid Supramolecular Complexes
}

\author{
Nadia Canilho, ${ }^{\dagger}$ Edis Kasëmi, ${ }^{\ddagger}$ A. Dieter Schliiter, ${ }^{\ddagger}$ Janne Ruokolainen, ${ }^{\S}$ and \\ Raffaele Mezzenga**,,
}

\author{
Department of Physics and Fribourg Center for Nanomaterials, University of Fribourg, \\ Ch. du Musée 3, CH-1700 Fribourg, Switzerland, Laboratory for Polymer Chemistry, \\ Swiss Federal Institute of Technology, Department of Materials, ETH-Zurich, \\ HCI J 541, CH-8093 Zurich, Switzerland, Helsinki University of Technology, \\ Physics Laboratory, Helsinki 02015, Finland, and Nestlé Research Center, \\ Vers-Chez-les-Blancs, 1000, Lausanne 26, Switzerland
}

\begin{abstract}
The present work describes the molecular structure and the topology in real space of self-assembled comblike liquid crystalline polymers based on supramolecular ionic complexation of dendronized polymers and lipids. The molecular organization was studied as a function of the generation of the dendronized polymers and the length of the lipid tails, both parameters being known to strongly influence the final liquid crystalline (LC) structures stable at thermodynamic equilibrium (Macromolecules 2007, 40, 2822-2830). Transmission electron microscopy (TEM) on selectively stained samples allowed imaging of the structures in real space, the identification of individual domains in the various LC phases, and direct comparison of lattice periods with values obtained by small-angle X-ray scattering (SAXS). TEM and SAXS were found in perfect agreement, and surprisingly, the alkyl tails were shown to form the discrete domains in all LC columnar phases. On the basis of these results, various possible molecular packing motifs in the LC phases were considered. First, the combination of density measurements of individual dendronized polymers and lipids with the ratio of lipid-dendronized polymer complexation, measured by elemental analysis, allowed establishing the exact volume fraction of the two supramolecular components and thus, the radii of cylinders and lamellae widths in the various columnar and lamellar LC phases observed. Then, by comparing these topological values with the contour length of the lipids, it was possible to conclude that, in all columnar phases, the lipids adopt a fully stretched configuration with homeotropic arrangement, while in the lamellar phase, lipids tails are essentially interdigitated. The control of lattice periods of LC structures in the range of $2-5 \mathrm{~nm}$ and the possibility to confine the lipids in the cylindrical domains make these materials as possible templates for nanoporous materials and ultra densely packed nanochannels.
\end{abstract}

\section{Introduction}

Supramolecular chemistry has gained remarkable attention during the last 20 years as a viable technique to design complex macromolecular systems based on noncovalent bonds. ${ }^{1,2}$ Various routes have been studied to complex different molecules together, exploiting intermolecular forces of various natures such as metal coordination, polar and hydrogen bonding, $\pi-\pi$ interactions, and ionic complexation. The metal coordination chemistry involves weak to strong interactions depending on the type of metal and ligand used. ${ }^{3}$ The main advantage of this route is that it offers a directional-bonding approach, for which by changing the architecture of the metal and ligand, both onedimensional coordination polymers ${ }^{4}$ or multidimensional macromolecular complexes can be formed..$^{5-7}$

Unidirectional $\pi-\pi$ interactions, which are characterized by medium-strong binding energies, have also been extensively

\footnotetext{
* Corresponding author. Department of Physics and Fribourg Center for Nanomaterials, University of Fribourg, Pérolles, Fribourg, CH-1700, Switzerland. E-mail: raffaele.mezzenga@unifr.ch. Telephone: + 4126300 9066. Fax: + 4126300 9747. Nestlé Research Center, Vers-Chez-LesBlanc, 1000 Lausanne 26, Switzerland. E-mail: raffaele.mezzenga@rdls. nestle.com. Telephone: + 4121785 8078. Fax: + 41217858554.

†epartment of Physics and Fribourg Center for Nanomaterials, University of Fribourg.

Laboratory for Polymer Chemistry, Swiss Federal Institute of Technology.

$\S$ Helsinki University of Technology, Physics Laboratory.

"Nestlé Research Center.
}

used to self-assemble small oligomers into discotic liquid crystals with functional properties, such as increased charge mobility. ${ }^{8-11}$ More recently, such interactions have been used to assist self-assembly of $\pi$-conjugated rod-coil block copolymers into suitable morphologies for possible use in photovoltaic applications. $^{12}$

Hydrogen bonding is characterized by small binding energy compared to other kinds of bonding in supramolecular assemblies, which has allowed the design of comblike polymers with temperature-responsive behavior based on homopolymers with hydrogen-bonding accepting sites and hydrogen-bonding donor surfactants. ${ }^{13,14}$ The binding properties in hydrogenbonded assemblies can be further tuned by increasing the number of hydrogen bond acceptor-donor pairs between the substrate and the ligand. ${ }^{15}$ This has as major effect to increase the binding energy and to provide molecular recognition mechanisms. ${ }^{16}$

Ionic complexation is in many respects similar to hydrogen bonding, but the binding energy is sensibly higher. This is especially relevant in biological phenomena such as coacervation and complexation of biological polyelectrolytes and phospholipids ${ }^{17-19}$ and can be used to design $\mathrm{pH}$-responsive materials. With synthetic compounds, this route has been widely used to design liquid crystalline polymers with coiled, ${ }^{20} \operatorname{rod},{ }^{21}$ dendritic, ${ }^{22}$ dendronized backbones, ${ }^{23-29}$ and metallosupramolecular polyelectrolytes. ${ }^{30,31}$ The liquid crystallinity is provided by the microphase separation driven by the segregation between 

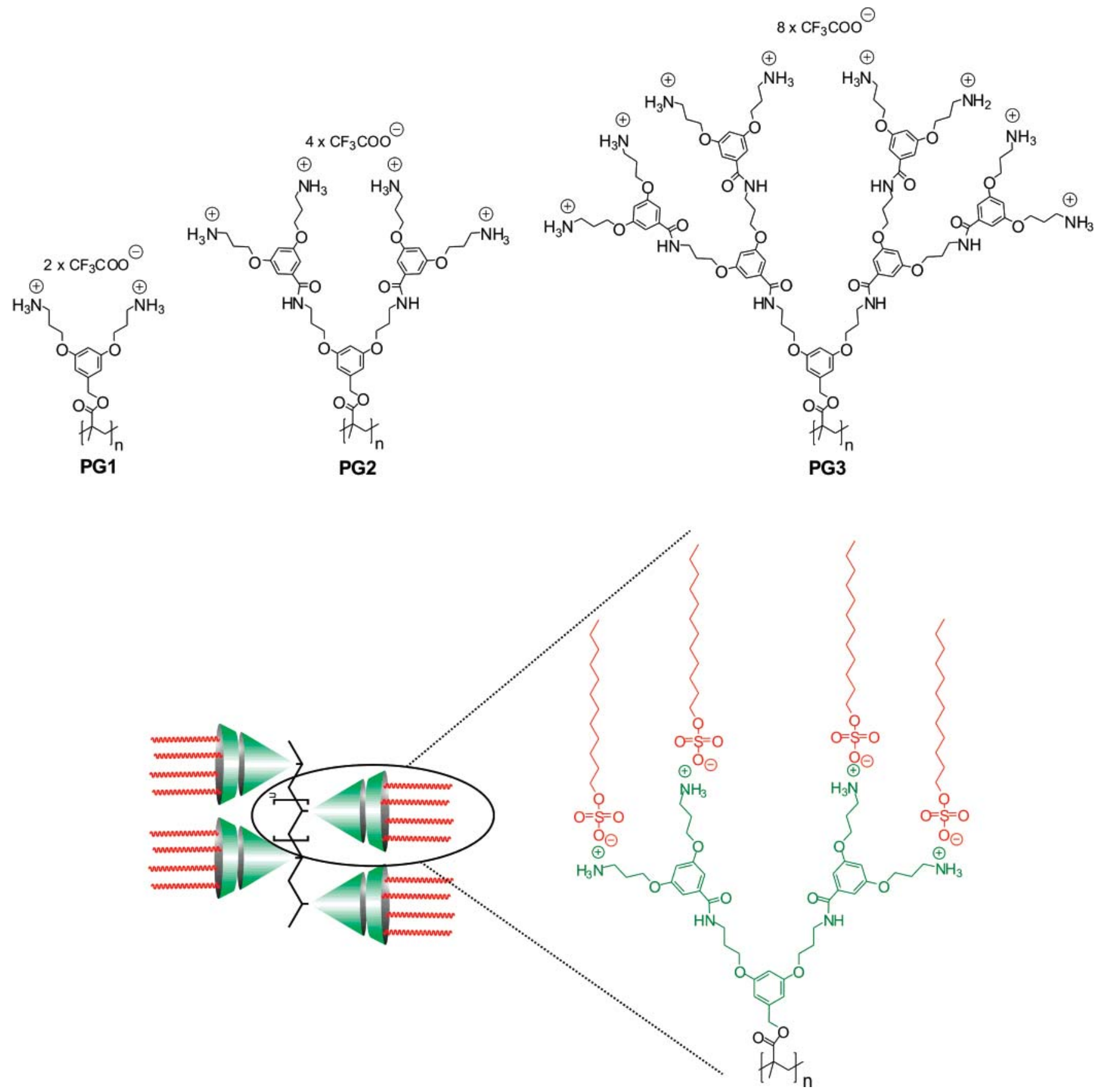

Figure 1. Chemical structures of dendronized polymers PG1, PG2, PG3, and schematic representation of the ionic complex.

Table 1. Data from Elemental Analysis of all Dendronized Polymer-Surfactant Complexes Investigated

\begin{tabular}{|c|c|c|c|c|c|c|}
\hline entry & complex & $\mathrm{C}$ expected/found & $\mathrm{H}$ expected/found & $\mathrm{N}$ expected/found & S expected/found & $\mathrm{Cl}$ expected/found \\
\hline 1 & PG1-C8 & $53.35 / 53.42$ & $8.41 / 8.31$ & $3.77 / 3.78$ & $8.63 / 8.55$ & \\
\hline 2 & PG2-C8 & $54.13 / 53.99$ & $8.12 / 8.09$ & $5.05 / 5.00$ & $7.71 / 7.58$ & \\
\hline 3 & PG3-C8 & $54.46 / 54.18$ & 7.99/7.73 & $5.59 / 5.59$ & $7.32 / 7.02$ & \\
\hline 4 & PG1-C12 & $57.58 / 57.33$ & $9.19 / 9.13$ & $3.28 / 3.29$ & $7.50 / 7.27$ & $0.00 / 0.05$ \\
\hline 5 & PG2-C12 & $57.87 / 56.80$ & $8.86 / 8.49$ & $4.45 / 4.34$ & $6.79 / 7.07$ & $0.00 / 0.16$ \\
\hline 6 & PG2-C12 $(66 \%)$ & $54.93 / 57.83$ & $7.78 / 8.98$ & $5.07 / 4.44$ & $4.79 / 6.91$ & $0.00 / 0.19$ \\
\hline 7 & PG2-C12 $(200 \%)$ & $54.88 / 58.07$ & $8.81 / 8.86$ & $2.76 / 4.42$ & $8.43 / 6.84$ & $0.00 / 0.14$ \\
\hline 10 & PG2-C14 & $59.43 / 59.94$ & $9.17 / 9.28$ & $4.20 / 4.00$ & $6.41 / 6.37$ & \\
\hline 11 & PG3-C14 & $59.48 / 59.75$ & $9.02 / 8.86$ & $4.69 / 4.54$ & $6.14 / 6.23$ & \\
\hline 12 & PG1-C18 & $62.20 / 61.43$ & $10.04 / 9.87$ & $2.74 / 2.53$ & $6.27 / 5.98$ & \\
\hline 13 & PG2-C18 & $62.07 / 63.25$ & $9.69 / 9.79$ & $3.78 / 3.32$ & $5.76 / 5.33$ & \\
\hline 14 & PG3-C18 & $62.02 / 62.06$ & $9.54 / 9.56$ & $4.24 / 4.11$ & $5.54 / 5.42$ & \\
\hline
\end{tabular}

the polyelectrolyte, which acts as macromolecular template, and the counter-charged moieties. In the most common case of ionic hydrocarbon-based surfactant moieties, the typical length scale at which these liquid crystals are structured is in the range of
2-5 nm. ${ }^{32}$ The most reliable techniques used to characterize these structures are small- and wide-angle X-ray scattering, coupled with cross-polarized optical microscopy to distinguish among different birefringent behavior, as well as differential 


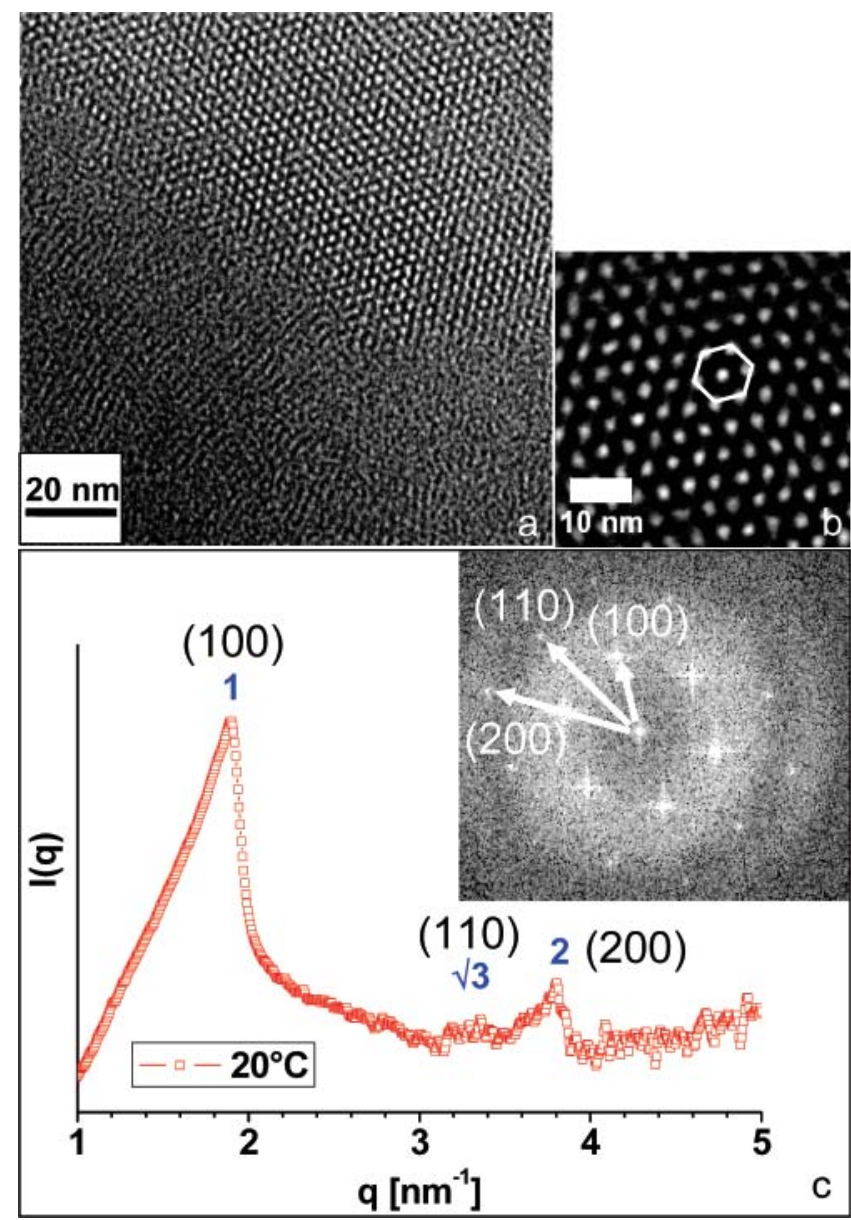

Figure 2. (a) Large magnification TEM picture observed for PG1C12 complex showing a long-range ordered columnar hexagonal liquid crystalline phase. (b) High magnification TEM picture of PG1-C12 columnar hexagonal phase. The window size is $50 \mathrm{~nm} \times 50 \mathrm{~nm}$ (c) Comparison of SAXS diffractogram from ref 29 and FFT (inset) for PG1-C12 columnar hexagonal phase.

scanning calorimetry. On the other hand, the characterization of these supramolecular materials in the real space by microscopy techniques such as transmission electron microscopy (TEM) and atomic force microscopy (AFM) has been severely hindered by the very small characteristic feature sizes and the very weak contrast between the domains. As a major consequence, although lattice parameter can be accurately determined by scattering techniques, the exact topology and molecular organization cannot be univocally established by relying on scattering data only, and assumptions on which component is actually forming the individual domains have to be made in order to propose tentative molecular packing model for both the polymer and the ionically bound mesogenes.

Yet, the exact identification of individual domains is essential when specific applications have to be targeted. For example, Ikkala et al. have developed hierarchical structures based on comblike block copolymers and surfactants in which the surfactant is first confined into hexagonally packed cylinders and successively removed by a selective solvent to yield mesoporous materials. ${ }^{33}$ Similar concepts can be directly applied to ionically bound liquid crystalline polymers where, however, considering the smaller characteristic length scales, nanoporous material with channel density well beyond that reported for block copolymer systems can be designed. ${ }^{34}$

In previous work, we have reported the use of cationic dendronized polymers and anionic lipids as a viable system to design liquid crystalline polymers spanning a rich phase diagram
Table 2. Lattice Parameters and Structures for the Complexes Investigated

\begin{tabular}{lll}
\hline & lattice parameters, $\mathrm{nm}$ & LC packing \\
\hline PG1-C8 & & amorphous \\
PG1-C12 & $a=3.85$ & hexagonal \\
PG1-C14 & $a=4.83$ & hexagonal \\
PG1-C18 & $d=4.22$ & lamellar \\
PG2-C8 & $a=3.69 ; b=2.41$ & rectangular \\
PG2-C12 & $a=3.92$ & tetragonal \\
PG2-C14 & $a=4.18$ & tetragonal \\
PG2-C18 & $d=4.62$ & lamellar \\
PG3-C8 & $a=5.23 ; b=2.96$ & rectangular \\
PG3-C12 & $a=4.72$ & tetragonal \\
PG3-C14 & $a=4.78$ & tetragonal
\end{tabular}

composed of columnar rectangular, hexagonal, tetragonal, and lamellar phases, all organized in the range of $2-5 \mathrm{~nm}^{28,29}$ The exact topology of each phase, however, such as the identification of individual domains, volume fractions, radii of cylinders, or widths of lamellar layers, were not univocally established by microscopy due to the challenging length scales involved.

With the perspectives of using dendronized polymer-lipid complexes as templates for functional nanoporous materials with well-defined lattices, in this paper, we present a real space characterization of the various dendronized polymer-lipid LC phases based on TEM analysis, with the identification of cylinders and continuous phase in all the columnar phases. These results are first compared with small-angle X-ray scattering data and then completed by the evaluation of individual domains volume fractions via elemental analysis and density measurements. Not only has this study provided important information on the nature of the various domains and the diameter of cylinders in the different columnar phases but also made possible the understanding of the molecular packing for the lipid tails in the LC phase considered.

\section{Experimental Section}

Materials and Complex Preparation. Four different anionic sulfated surfactant salts were used for ionic complexation with cationic dendronized polymers: sodium octyl sulfate $(\mathbf{C 8})$ (Fluka, 95\%), sodium dodecyl sulfate (C12, Fluka, 98\%), sodium tetradecyl sulfate $(\mathbf{C 1 4}$, Fluka, 95\%), and sodium octadecyl sulfate $(\mathbf{C 1 8})$ (Fluka, 98\%).

First (PG1), second (PG2), and third generation (PG3) cationic dendronized polymers, ${ }^{35-37}$ carrying respectively 2,4 , and 8 positively charged ammonium groups per repeat unit, were selected to form comblike ionic supramolecular complexes as schematically sketched in Figure 1. To study the effect of the initial positivenegative charges ratio on the structure and composition of the final complexes, the stoichiometric ratio of negative sulfate:positive ammonium charges was systematically varied by a factor 2,1 , and 0.66 , respectively. Complexation was realized in water solutions maintained at $\mathrm{pH}=3-4$ in order to retain the positive and negative charges of dendronized polymers and surfactants, respectively. Lyophilized dendronized polymers were dissolved into a primary water solution under continuous stirring. Then a secondary water solution at the same $\mathrm{pH}$, containing the equivalent molar mass of surfactant needed to access the targeted positive-negative charges ratio, was added dropwise to the first solution under continuous stirring. As a result of the ionic complexation between polymers and surfactants, the solution progressively became turbid, and then the complex was centrifuged and collected, then dried under vacuum at room temperature for 3 days, and finally available for chemical and physical characterization. More details on the ionic complexation procedure between dendronized polymers and surfactants can be found in a previous publication. ${ }^{29}$

Elemental Analysis. Elemental analyses were performed on a Leco CHN-900 and Leco CHNS-932 instrument using acetanilide/ cholesterol, atropine, and caffeine as reference for calibration. For 


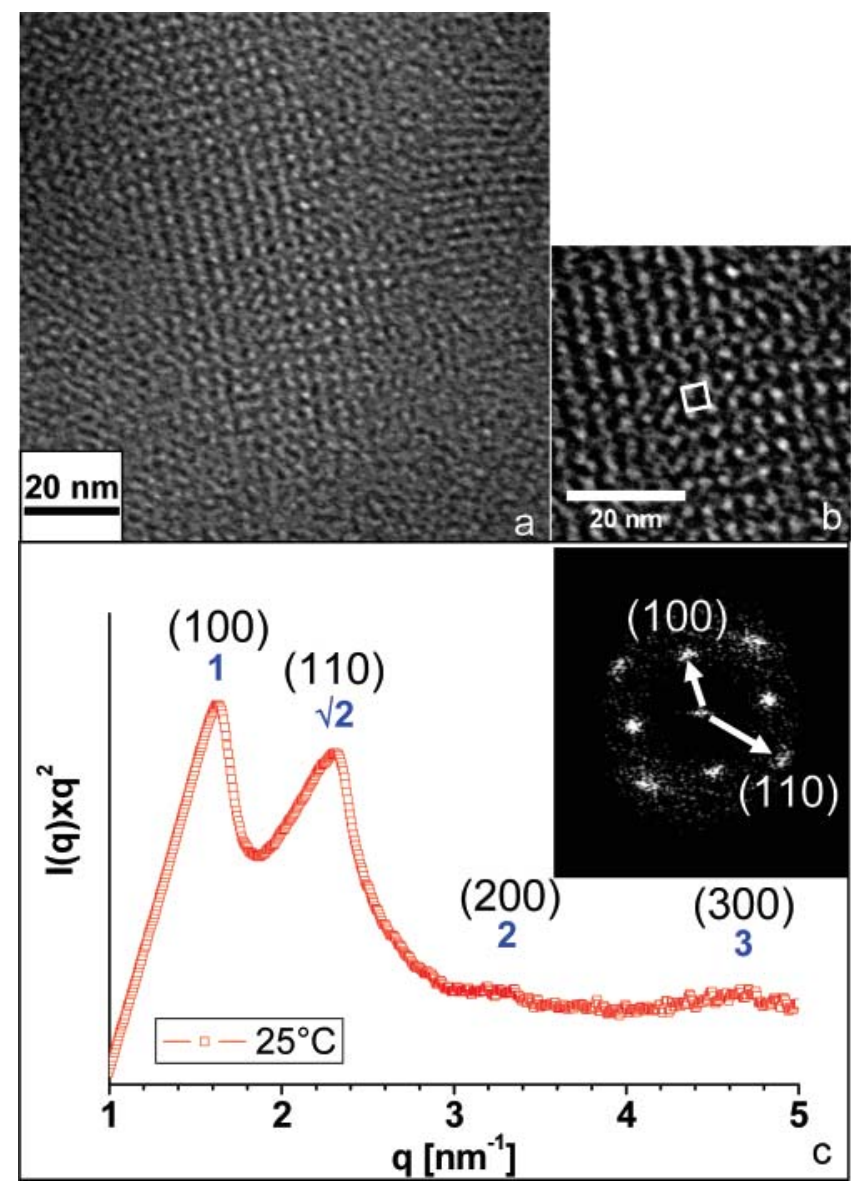

Figure 3. (a) Large magnification TEM picture observed for PG2C12 complex showing a long-range ordered columnar tetragonal liquid crystalline phase. (b) High magnification TEM picture of PG2-C12 columnar tetragonal phase. The window size is $50 \mathrm{~nm} \times 50 \mathrm{~nm}$. (c) Comparison of SAXS diffractogram from ref 29 and FFT (inset) for PG2-C12 columnar tetragonal phase.

$\mathrm{Cl}$ determination, $5 \mathrm{mM}$ of $\mathrm{AgClO}_{4}$ were used as a reagent. The samples were dried rigorously under vacuum prior to analysis to remove strongly adhering solvent molecules.

Density Measurements. The helium pycnometer used was a Accupyc 1330 from Micromeritics. The measurement method is based on Archimede's principle of fluid displacement and Boyle's law to determine the envelope volume of a solid.

At first, a reference was made with the empty cell. Then ca. 2 $\mathrm{g}$ of polymer at room temperature were introduced in the cell for measurements of the volume. Twenty cycles of measurements were acquired to yield accurate estimations of densities, and values reported are based on averages out of these series of measurements.

Annealing Procedure. The protocol of thermal annealing procedure yielding thermodynamically stable structures was as follows: the complexes were annealed for 3 days in a high vacuum column $\left(10^{-8} \mathrm{mBar}\right)$ at temperatures depending on the specific complex considered (typically between 80 and $150{ }^{\circ} \mathrm{C}$ ) but always comprised between the glass transition temperature of the dendronized polymers $\left(T_{\mathrm{g}}\right)$ and the melting temperature of the lipids $\left(T_{\mathrm{m}}\right)$ and the temperature of order-disorder transition $\left(T_{\mathrm{ODT}}\right)$ of the specific liquid crystalline structure, as measured by separate temperature-dependent SAXS experiments. The achievement of thermodynamic equilibrium structures was demonstrated by the fact that samples shortly processed at temperatures above the $T_{\text {ODT }}$ into the isotropic fluid region (for $12 \mathrm{~h}$ ), and then annealed below the $T_{\mathrm{ODT}}$, recovered structures identical to those observed directly after 3 days annealing at temperatures $T_{\mathrm{g}}<T<T_{\mathrm{ODT}}$.

Small-Angle X-ray Scattering. Small- and wide-angle X-rays scattering (SWAXS) experiments were performed on a SAXSess instrument using a $\mathrm{Cu} \mathrm{K} \alpha(\lambda=0.1542 \mathrm{~nm}) \mathrm{X}$-ray source and a slit camera configuration. Temperature of samples was controlled in the $10-200{ }^{\circ} \mathrm{C}$ range. Powder samples were placed in between two mica foils within the sample holder, and diffraction data were acquired during $30 \mathrm{~min}$ exposure. Each diffractogram was acquired after $1 \mathrm{~h}$ time equilibration at isothermal temperatures. Mica background signal was removed from measured diffractograms, yielding the final SWAXS data. Measurements acquired following this protocol were shown to be highly reproducible.

Transmission Electron Microscopy. For transmission electron microscopy, (TEM) ultrathin sections (approximately 40-70 nm) were cryomicrotomed at $T=-60{ }^{\circ} \mathrm{C}$ by using a Leica Ultracut UCT ultramicrotome and a Diatome diamond knife. Sections were picked up onto 400 mesh lacey or holey carbon-film-coated copper grids and, in order to enhance contrast, the microtomed sections were stained for $20 \mathrm{~min}$ in vapors of aqueous $\mathrm{RuO}_{4}$ solution $(0.5 \%$ solution, Electron Microscopy Science), which stains the aromatic hydrocarbons, i.e., dendronized polymer domains. Bright-field TEM was performed on a FEI Tecnai 12 transmission electron microscope operating at an accelerating voltage of $120 \mathrm{kV}$. Images were recorded using Gatan Ultra-Scan 1000 camera with a chargecoupled device $(C C D)$ size of $2048 \times 2048$. The dominating contrast mechanism for these samples was the phase contrast, and all the images were taken at the optimum defocus values when the phase contrast reaches the maximum value. ${ }^{14,38}$

\section{Results and Discussion}

In a previous study, ${ }^{29}$ the degree of loading of PG3 with $\mathbf{C 1 2}$ surfactants was described. By applying ${ }^{1} \mathrm{H}$ and ${ }^{19} \mathrm{~F}$ NMR spectroscopy as well as elemental analysis, quantitative loadings were determined. This was based on (a) a near-correct integration in the ${ }^{1} \mathrm{H}$ NMR spectrum, (b) the fact that, in the ${ }^{19} \mathrm{~F}$ NMR spectrum, no indication of any residual trifluoroactetate (the initial dendronized polymer counterion) was found, and (c) the correct data from elemental analysis for the elements $\mathrm{C}, \mathrm{H}, \mathrm{N}$, $\mathrm{S}$, and $\mathrm{Cl}$. On the basis of these results, the present set of dendronized polymer-surfactant complexes was mainly investigated by elemental analysis. As can be seen from Table 1 (entries 1-5 and 8-14), all data match the ones as calculated by assuming 1:1 complexes. Moreover, selected ${ }^{19} \mathrm{~F}$ NMR spectra confirmed that there was no residual trifluoroacetate. In an attempt to produce a situation in which the coverage is not complete, PG2 was exposed to only $66 \%$ of the required C12 surfactant. After isolation of the complex (in a relatively low yield of $25 \%$ ), its elemental analysis was performed (Table 1 , entry 6). As can be seen, the values clearly support a 1:1 coverage despite this mismatch. The same was obtained if the surfactant was used in an excess of $100 \%$ (Table 1, entry 7) except for the much higher yield in which the aggregate was obtained $(82 \%)$. This seemingly inconsistent observation finds its explanation in the fact that the dendronized polymersurfactant complexes precipitate from the reaction medium once a complete coverage is reached. This is on the one hand the driving force for a virtually full coverage (at the expense of a low yield) and on the other a "protection" against any incorporation of surfactant molecules that are not required for charge compensation. Also, for these latter two examples, ${ }^{19} \mathrm{~F}$ NMR spectra were recorded, which again did not show any relevant signals except for the internal integration standard $\left(\mathrm{C}_{6} \mathrm{~F}_{6}\right)$, which had been added in the nanomolar range from a stock solution with precision instruments.

The detailed assessment of the molecular architecture of the dendronized polymer-lipid complexes was the first necessary step toward investigation of their solid-state properties. To establish the structure and topology of the various LC phases obtained for the solid complexes at thermodynamic equilibrium and the influence of both polymer generation and lipid tail 

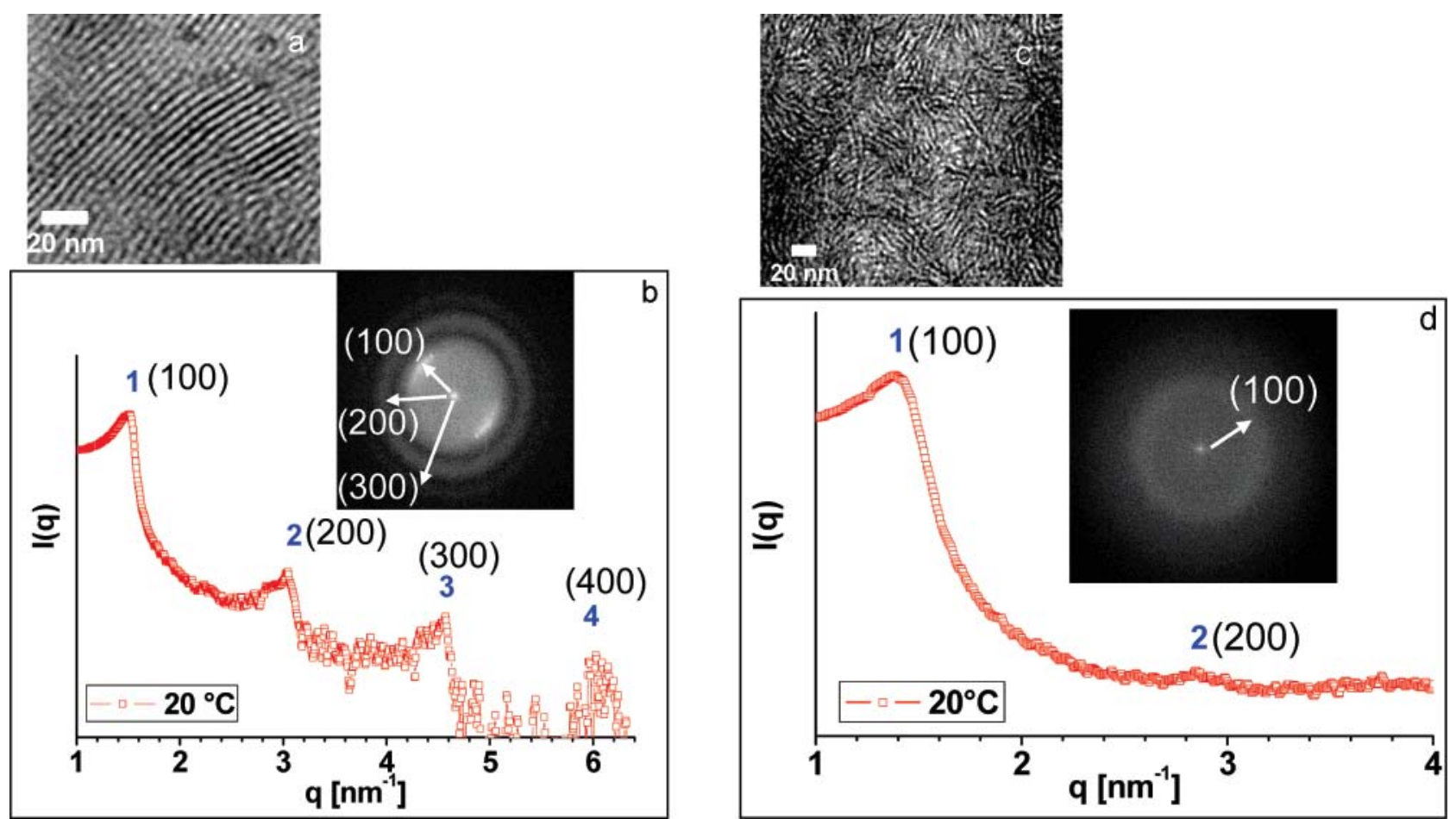

Figure 4. (a) Large magnification TEM picture of a long-range ordered lamellar liquid crystalline phase for PG1-C18. (b) Corresponding SAXS diffractogram from ref 29 compared to the respective FFT image (inset). (c) Large magnification TEM picture of a short-range ordered lamellar arrangement liquid crystalline phase for the complex PG2-C18. (d) Corresponding SAXS diffractogram from ref 29 compared to the respective FFT image (inset).

length, entries 1 to 14 in Table 1 were collected and investigated both in the reciprocal space by SAXS and in real space by TEM.

Detailed systematic SAXS characterization for the various dendronized polymer-lipid complexes was already discussed in a previous paper. ${ }^{29}$ The results and the determination of lattice parameters for various LC phases are summarized in Table 2.

Although the exact liquid crystalline group space was determined for all complexes by combining SAXS analysis, cross-polarized optical microscopy, and differential scanning calorimetry (DSC), the exact topology, composition, and characteristic sizes of cylindrical or lamellar domains could not be uniquely assessed, and as a consequence, the molecular packing could only be tentatively proposed.

To provide better insight into the above points, TEM analysis was pursued. In principle, this technique allows one to unambiguously attribute each microdomain to the specific compounds forming the supramolecular complexes by selective chemical staining of individual domains in ultramicrotomed sections. The characteristic length scales of the LC phases considered in the present work, typically in the range $2-5 \mathrm{~nm}$, were very demanding to clearly resolve individual domains, and chemical staining proved to be a critical step in the identification process. Several staining agents were tried, such as $\mathrm{OsO}_{4}, \mathrm{I}_{2}$, and $\mathrm{RuO}_{4}$. The latter agent provided the best contrast to electrons with respect to the different domains as it selectively stains the aromatic groups present in the dendrons of the polymers without chemically binding to the fully saturated alkyl tails of the surfactants. ${ }^{39}$ Furthermore, because ultramicrotomed $60 \mathrm{~nm}$ thick sections stained by $\mathrm{RuO}_{4}$ presented unchanged contrast to TEM after 6 months from the staining process, possible contrast to electrons provided by preferential diffusion of $\mathrm{RuO}_{4}$ within alkyl tail domains could be unambiguously ruled out in favor of the chemical staining of the aromatic groups present in the dendrons.

Parts $\mathrm{a}$ and $\mathrm{b}$ of Figure 2 present the low- and highmagnification TEM micrographs, respectively, of PG1-C12 complex after 3 days of thermal annealing. A long-range order columnar hexagonal phase is shown, with lattice period of 3.60 $\mathrm{nm}$ in very good agreement with SAXS studies $(3.85 \mathrm{~nm}$, see Table 2). The additional important information compared to diffraction studies, however, is that the dark, stained polymeric domains form the continuous phase, while the clear domains, which identify the unstained alkyl tails, form the cylinders.

As a further direct comparison of TEM results with diffraction data, the inset in Figure 2c presents the fast Fourier transform (FFT) of a PG1-C12 TEM micrograph. As in the case of corresponding SAXS diffractograms (Figure 2c), the first three order reflections corresponding to (100), (110), and (200) Miller indexes are visible, as labeled in the image.

Parts a and b of Figure 3 show low- and high-magnification micrographs, respectively, for the microphase-separated morphology in the PG2-C12 complex after 3 days of thermal annealing. A long-range ordered columnar tetragonal phase is observed, with lattice period of $4.10 \mathrm{~nm}$, again in very good agreement with SAXS studies (3.92 nm). To our knowledge, it is the first time that a tetragonal phase in polymeric systems is imaged in real space at these small length scales. Also, in this case, as for the columnar hexagonal phases, the clear domains identifying the unstained lipid component form the cylinders, while the dark stained polymer domains fill the continuous phase around the cylinders. For the purpose of direct comparison with SAXS and TEM analysis, the FFT of a PG2-C12 TEM micrograph (inset) is compared with the corresponding SAXS diffractogram in Figure 3c. Two reflections, labeled as (100) and (110), are visible in the FFT image of the columnar tetragonal phase, which identify with the first two out the four reflections visible in the corresponding SAXS diffractogram (Figure 3c). ${ }^{28,40}$

Parts $a-d$ of Figure 4 show the TEM micrographs and corresponding FFT for PG1-C18 and PG2-C18 complexes after 3 days annealing. In the case of PG1-C18 (Figure 4a), a 
Table 3. Summary of the Densities Values Measured by Helium Pycnometry in Solid Samples

\begin{tabular}{lc}
\hline & density $\left[\mathrm{g} / \mathrm{cm}^{3}\right]$ \\
\hline PG1 & 1.446 \\
PG2 & 1.480 \\
PG3 & 1.464 \\
C8 & 1.052 \\
C12 & 1.090 \\
C18 & 1.128 \\
\end{tabular}

Table 4. Surfactant Volume Fractions Calculated for Each Complex, Based on Density Measurements and Elemental Analysis

\begin{tabular}{lcccc}
\hline & C8 & C12 & C14 & C18 \\
\hline PG1 & 0.57 & 0.62 & 0.63 & 0.67 \\
PG2 & 0.50 & 0.55 & 0.57 & 0.61 \\
PG3 & 0.47 & 0.52 & 0.54 & 0.58
\end{tabular}

Table 5. Calculated Radii ${ }^{a}$ of Columnar Phases $(r, \mathrm{~nm})$ and Widths of Surfactant Lamellar Layers Distances ${ }^{b}(d, \mathbf{n m})$

\begin{tabular}{lcccc}
\hline & $\mathbf{C 8}^{a}$ & $\mathbf{C 1 2}^{a}$ & $\mathbf{C 1 4}^{a}$ & $\mathbf{C 1 8}^{b}$ \\
\hline PG1 & & 1.59 & 2.06 & 2.81 \\
PG2 & 1.21 & 1.65 & 1.82 & 2.79 \\
PG3 & 1.55 & 1.92 & 2.03 &
\end{tabular}

well-ordered lamellar phase can be observed, as also reflected by the FFT image (inset Figure 4b), in which 3 reflections can be identified corresponding to (100), (200), and (300). This agrees well with SAXS diffractograms of PG1-C18, which exhibit four consecutive reflections indicative of long-range order (see Figure 4b). The period of the lamellar phase based on TEM images is measured at $4.20 \mathrm{~nm}$, which again is in perfect agreement with the SAXS data of $4.2 \mathrm{~nm}$. In the case of PG2-C18 (Figure 4c), the morphology is still lamellar with a $4.80 \mathrm{~nm}$ period, but long-range order is lost, as also revealed by the FFT image, which presents only one reflection (inset Figure 4d). This is consistent with the SAXS diffraction data, which for PG2-C18 show a first strong peak centered at $q_{1}=$ $1.36 \mathrm{~nm}^{-1}$ corresponding to $4.6 \mathrm{~nm}$, followed by a very weak second-order reflection at $q_{1}=2.72 \mathrm{~nm}^{-1}$ (see Figure $4 \mathrm{~d}$ ).

Because Figures 2-4 report TEM images acquired out of focus to provide sufficient contrast among different domains, the volume fraction of lipid-polymer domains in the micrographs are either over- or underestimated by an amount that is directly proportional to the distance between the image acquisition plane and the focus plane. Thus, TEM cannot be used to quantitatively appraise the volume fraction of clear and dark domains, and alternative methods were necessary to assess the real volume fraction of lipid and dendronized polymer in the various complexes. As will be shown in what follows, however, these values can be determined by measuring the individual densities of the polymer and the lipid components.

Indeed, because elemental analysis has demonstrated a virtually equivalent ammonium-sulfate ratio in all the complexes studied, once densities of lipids and dendronized polymers are known, the volume fraction of surfactant $i$ in the complex formed with dendronized polymer of generation $j$ is given directly by:

$$
\phi_{i j}=\frac{\frac{2^{j} M_{i}}{\rho_{i}}}{\frac{M j}{\rho_{j}}+\frac{2^{j} M_{i}}{\rho_{i}}}
$$

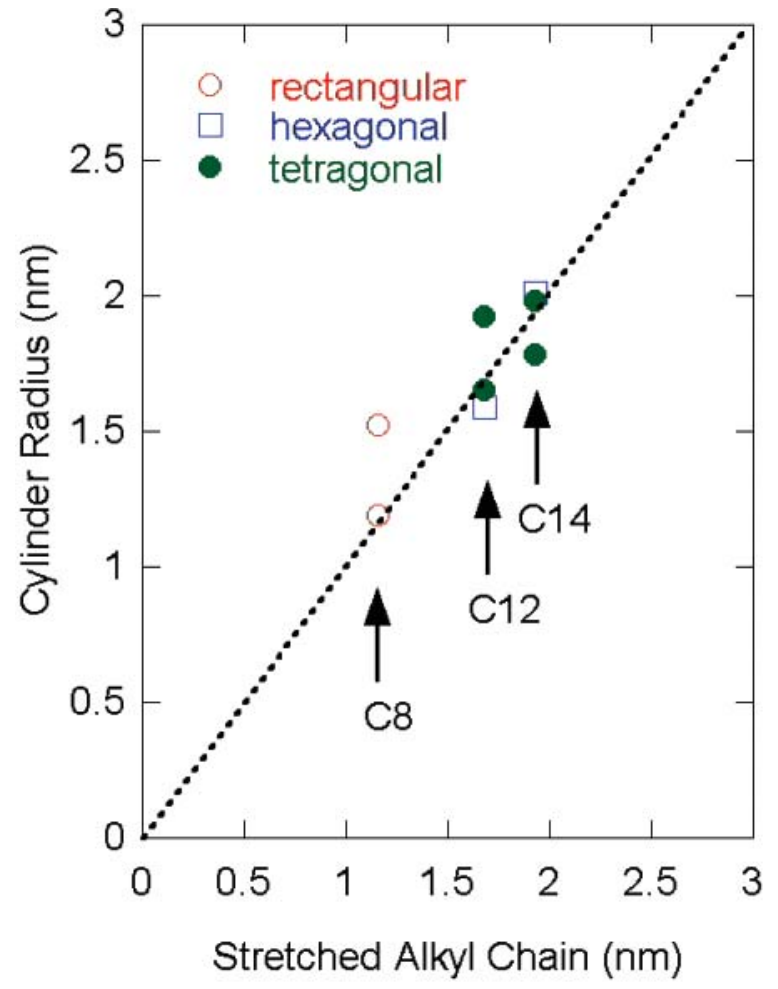

Figure 5. Comparison of the radii of the cylindrical dispersed surfactant phase with the theoretical surfactant stretched chain lengths for the various columnar phases observed.

because $2^{j}$ surfactant molecules of mass $M_{i}$ and density $\rho_{i}$ are attached to each repeat unit of the dendronized polymer having mass $M_{j}$ and density $\rho_{j}$.

The helium pycnometer method yield the densities of the surfactant and the dendronized polymer inclusive of their counter-charged ions, while $\rho_{i}$ and $\rho_{j}$ in eq 1 represent the densities values of surfactant and dendronized polymers without $\mathrm{Na}^{+}$and $\mathrm{CF}_{3} \mathrm{COO}^{-}$, respectively. In the present work, it was assumed that the measured densities and $\rho_{i}$ and $\rho_{j}$ are essentially the same, which represents only a minimum deviation from actual $\rho_{i}$ and $\rho_{j}$ values. Furthermore, gas pycnometry was preferred to measure $\rho_{i}$ and $\rho_{j}$ because this technique allows measuring the densities of both surfactant and polymer directly in the bulk state, thus avoiding possible density changes in solution of either component (see Table 3).

Table 4 reports the volume fractions of surfactants obtained for the various complexes based on eq 1. A few points deserve some discussion.

The first trend that becomes apparent when looking at Table 4 is that any possible rational on driving forces toward the stabilization of a specific LC structure cannot be based uniquely on volume fraction but rather has to account for both the effect of polymer generation and alkyl tail length. For example PG1C14 and PG1-C18 have similar volume fractions of lipid (0.63 and 0.67 , respectively) and are both based on the first generation of dendronized polymer. However, an increase in the length of the alkyl tail by four carbon atoms is sufficient to drive the columnar hexagonal phase into a long-range ordered lamellar phase. Similar considerations can be made for the PG2-C14 and PG2-C18 complexes, where an order-order transition is found between a columnar tetragonal and a lamellar phase. When going from a PG1-C12 to a PG2-C14 complex, the variations in both the length of the alkyl tail ( 2 carbon units) and lipid volume fraction are both small, but the change from 


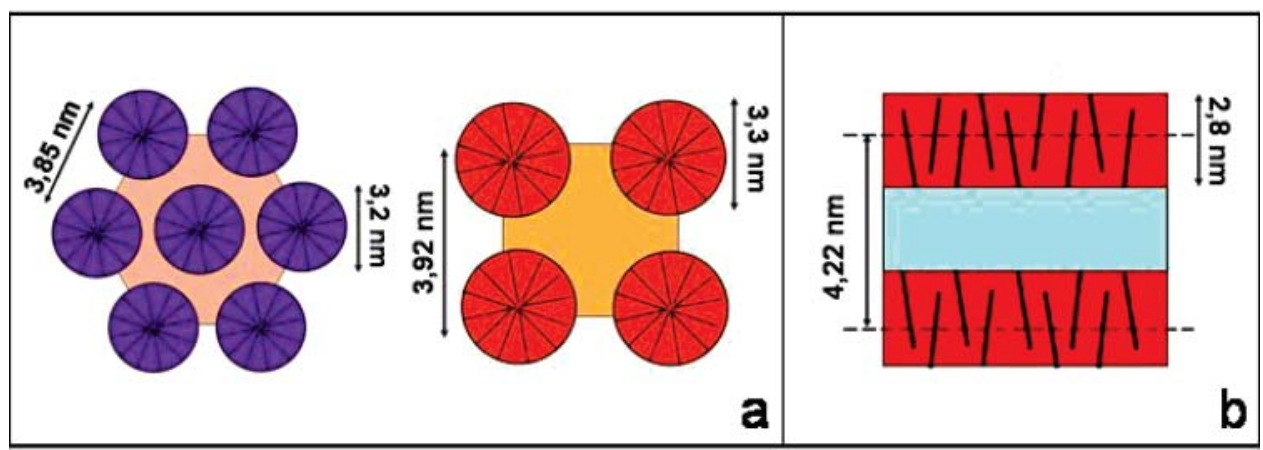

Figure 6. (a) Schematic representation of the packing mechanisms for the surfactant tails in the cylinders of columnar hexagonal (PG1-C12) and tetragonal (PG2-C12) phases. (b) Packing mechanisms for the surfactant tails in the lamellar phases (PG1-C18, PG2-C18).

a first- to a second-generation dendronized polymer is sufficient to induce a change from a columnar hexagonal to a columnar tetragonal LC phase. Tetragonal phases being observed only for second- and third-generation dendronized polymers, but with the same surfactants used to stabilize columnar hexagonal phases (C12 and C14), they are then possibly stabilized by the specific packing of dendrons rather than by specific lipid length or corresponding volume fractions.

The second, more unexpected result when observing the volume fractions of the lipids in the complexes reported in Table 4 is that the lipids constitute the majority phase in all the columnar phases while forming the cylinder domains. This represents a surprising result because such a configuration, although possible, does not minimize the interfacial energy associated with the lipid-polymer interface. This demonstrates that not only enthalpy energetic contributions but also entropic components such as the conformational entropy of the polymer backbone and dendrons must play a role in the minimization of the total free energy of the system at equilibrium.

Because the volume fractions of lipid and polymer components are known and individual domains have been identified, the radius of cylinders in the columnar phases, $r$, and width of lipid lamellae in the lamellar phase, $d$, can be calculated by the following simple expressions:

(i) For columnar rectangular phases:

$$
r=\sqrt{\frac{a b}{\pi} \phi_{i j}}
$$

with $a$ and $b$ being the longer and shorter rectangular lattice parameters.

(ii) For columnar tetragonal phases:

$$
r=a \sqrt{\frac{\phi_{i j}}{\pi}}
$$

with $a$ being the square lattice parameter.

(iii) For columnar hexagonal phases:

$$
r=a \sqrt{\frac{\sqrt{3}}{2} \frac{\phi_{i j}}{\pi}}
$$

with $a$ being the hexagonal lattice parameter.

(iv) For lamellar phases:

$$
d=a \phi_{i j}
$$

where $a$ is the lamellar period.

Table 5 shows, in nm, the typical sizes $r$ and $d$ obtained for the various dendronized polymer-lipid complexes obtained by eqs $2-5$.
The packing mechanisms of side mesogenes into cylinders have been already discussed in literature for comblike block copolymers with rigid mesogenes covalently attached to the main linear polymer backbone of one of the blocks. ${ }^{41}$ Two possible mechanisms were postulated, the homogeneous configuration in which mesogenes orient parallel to cylinders axis, and the homeotropic configuration, in which mesogenes orient orthogonally to cylinders axis, radially converging toward the central axis of the cylinders. In the present case, supposing the lipid tails as rigid mesogenic units, three main configurations can be postulated for the packing of lipid tails: (i) homogeneous configuration, with tails aligned along cylinder axis, (ii) homeotropic configuration, and (iii) tilted configuration with tails converging toward the central axis of the cylinders, but with a tilted angle with respect to cylinder axis.

However, because lipid chains are not pure rigid rods, they can also adopt configurations close to a random walk, thus filling the cylinders as a pure polymer melt would do. The most efficient packing mechanism will mostly depend on the driving force to segregate between the lipid and dendronized polymers, strong segregation being expected to favor stretched chains.

Figure 5 compares the calculated radii of the cylinders in the various LC columnar phases with the contour length of the lipid used to form the corresponding complexes. As it can be noted, within $3 \AA$ tolerance, all the points fall on the same master line, indicating that the radius of the cylinders is equal to the fully stretched alkyl tail independently of the particular surfactant or columnar LC phase considered.

This suggests that (i) the alkyl tails of the lipid chains are fully stretched and that (ii) the chains pack in a homeotropic configuration, both trends being expected to be the result of a very strong segregation between the main polymer backbone and the side lipid chains. Figure $6 a$ sketches the molecular organization of lipid alkyl tails in the columnar LC phases observed for the various dendronized polymer-lipid complexes.

In the case of the PG1-C18 and PG2-C18 lamellar phases (Figure 6b), although the increase of lattice parameter $a$ goes from 4.2 to $4.6 \mathrm{~nm}$ when increasing generation from PG1 to PG2, the width of the lipid lamellae is calculated by eq 5 to remain constant at $2.8 \mathrm{~nm}$. Thus the increase in lattice parameter is due uniquely to the increase of generation, while the thickness of the lipid layer is unchanged, in agreement with the constant length of the lipid in the two lamellar structures. Furthermore, by comparing the $2.8 \mathrm{~nm}$ to the $2.5 \mathrm{~nm}$ contour length of fully stretched C18 sulfate lipids, an almost complete interdigitated configuration of the alkyl tails within the lipid domains can be expected.

In summary, in the present work, we have provided experimental evidence that in dendronized polymer-lipid ionic supramolecular complexes, the surfactant tails can be confined 
into discrete cylindrical domains despite the fact that these account for the majority volume fraction. By using suitable processes to remove the lipid phase, while preserving the dendronized polymers (such as increases of $\mathrm{pH}$ to enough basic values leading to deprotonation of the ammonium charges in the dendronized polymers), these liquid crystalline phases can potentially be used to design nanoporous material with large interfaces and controllable pore sizes.

\section{Conclusions}

We have investigated the complexation efficiency and real space structures of liquid crystalline phases formed by dendronized polymer-lipid ionic complexes by means of elemental analysis, density measurements, and transmission electron microscopy. Elemental analysis allowed demonstration that, for every generation of dendronized polymer and lipid length, an equimolar charges complexation occurs independently of the initial stoichiometric ratio used. This demonstrates that the complex precipitates from water only when a 1:1 complexation is achieved and that any eventual excess component (either surfactant or polymer) remains in solution. Selective staining of the dendronized polymers by $\mathrm{RuO}_{4}$ allowed identifying both the lipid and polymer domains in both columnar and lamellar phases. It was then surprisingly found that, although lipid surfactants form the domains with the majority volume fraction, they are confined within the cylinder domains in all the columnar phases, e.g., rectangular, hexagonal, and tetragonal columnar phases, respectively. Indeed, by measuring the individual densities of both surfactants and polymers, volume fractions of lipid and polymeric domains could be established in all the various liquid crystalline phases, and the thickness of lamellar layers or cylinders radii in lamellar and columnar phases, respectively, could then be derived by simple topological models.

To provide a rational for the molecular packing of the lipids in the various structures, the characteristic size of lipid domains was then compared with the length of fully stretched lipid alkyl tails. It was shown that, for lamellar phases, lipids essentially pack with interdigitated alkyl chains, while in the case of columnar phases, the packing of alkyl tail is expected to occur in an homeotropic manner in which the chains are fully stretched and oriented radially and orthogonally with respect to the cylinder axis. These results were understood in terms of the strong segregation driving microphase separation of polymer and lipid domains and the conformational entropy of dendrons and are anticipated to have an impact in the design of organic nanoporous systems.

Acknowledgment. We are grateful to the Swiss National Science Foundation for financial support. We cordially thank Dr. H. Rueegger, M. Schneider, and M. Colussi, all at ETHZ, for their competent help with NMR spectroscopy, elemental analysis and GPC, DSC, and TGA measurements, respectively. We are also grateful to M. Morais from the Powder Technology Laboratory at EPFL for his contribution in the pycnometer measurements.

\section{References and Notes}

(1) Suárez, M.; Lehn, J. M.; Zimmerman, S. C.; Skoulios, A.; Heinrich, B. J. Am. Chem. Soc. 1998, 120, 9526-9532.

(2) Kato, T.; Mizoshita, N.; Kishimoto, K. Angew. Chem., Int. Ed. 2006, $45,38-68$

(3) Holliday, B. J.; Mirkin, C. A. Angew. Chem., Int. Ed. 2001, 40, $2022-$ 2043.
(4) Khlobystov, A. N.; Blake, A. J.; Champness, N. R.; Lemenovskii, D A.; Majouga, A. G.; Zyk, N. V.; Schroder, M. Coord. Chem. Rev. 2001, 222, 155-192.

(5) Leininger, S.; Olenyuk, B.; Stang, P. J. Chem. Rev. 2000, 100, 853907.

(6) Andreopoulou, A. K.; Kallitsis, J. K. Eur. J. Org. Chem. 2005, 44484458.

(7) Tzanetos, N. P.; Andreopoulou, A. K.; Kallitsis, J. K. J. Polym. Sci., Polym. Chem. 2005, 43, 4838-4848.

(8) Struijk, C. W.; Sieval, A. B.; Dakhorst, J. E. J.; van Dijk, M.; Kimkes, P.; Koehorst, R. B. M.; Donker, H.; Schaafsma, T. J.; Picken, S. J.; van de Craats, A. M.; Warman, J. M.; Zuilhof, H.; Sudhölter, E. J. R. J. Am. Chem. Soc. 2000, 122, 11057-11066.

(9) Fechtenkötter, A.; Tchebotareva, N.; Watson, M.; Mullen, K. Tetrahedron 2001, 57, 3769-3783.

(10) Nguyen, T. Q.; Martel, R.; Avouris, P.; Bushey, M. L.; Brus, L.; Nuckolls, C. J. Am. Chem. Soc. 2004, 126, 5234-5242.

(11) Würthner, F. Chem. Commun. 2004, 1564-1579.

(12) Sary, N.; Mezzenga, R.; Brochon, C.; Hadziioannou, G.; Ruokolainen, J. Macromolecules 2007, 40, 3277-3286.

(13) Kato, T.; Fréchet, J. M. J.; Wilson, P. G.; Saito, T.; Uryu, T.; Fujishima, A.; Jin, C.; Kaneuchi, F. Chem. Mater. 1993, 5, 1094-1100.

(14) Ruokolainen, J.; Tanner, J.; Ikkala, O.; ten Brinke, G.; Thomas, E. L. Macromolecules 1998, 31, 3532-3536.

(15) Valkama, S.; Ruotsalainen, T.; Nykänen, A.; Laiho, A.; Kosonen, H.; ten Brinke, G.; Ikkala, O.; Ruokolainen, J. Macromolecules 2006, 39, 9327-9336.

(16) Sijbesma, R. P.; Beijer, F. H.; Brunsveld, L.; Folmer, B. J. B.; Hirschberg, J.; Lange, R. F. M.; Lowe, J. K. L.; Meijer, E. W. Science 1997, 278, 1601-1604.

(17) Rädler, J. O.; Koltover, I.; Salditt, T.; Safinya, C. R. Science 1997, 275, 810-814.

(18) Koltover, I.; Salditt, T.; Rädler, J. O.; Safinya, C. R. Science 1998 , $281,78-81$

(19) Salditt, T.; Koltover, I.; Rädler, J. O.; Safinya, C. R. Phys. Rev. E 1998, 58, 889-904.

(20) Antonietti, M.; Conrad, J.; Thünemann, A. Macromolecules 1994, 27, 6007-6011.

(21) Ponomarenko, E. A.; Waddon, A. J.; Bakeev, K. N.; Tirrell, D. A.; MacKnight, W. J. Macromolecules 1996, 29, 4340-4345.

(22) Marcos, M.; Martín-Rapún, R.; Omenat, A.; Barberá, J.; Serrano, J. L. Chem. Mater. 2006, 18, 1206-1212.

(23) Schlüter, A. D.; Rabe, J. P. Angew. Chem., Int. Ed. 2000, 39, 864883.

(24) Zhang, A. F.; Shu, L. J.; Bo, Z. S.; Schlüter, A. D. Macromol. Chem. Phys. 2003, 204, 328-339.

(25) Schlüter, A. D. In Functional Molecular Nanostructutres; Topics in Current Chemistry, Vol. 245; Springer: New York, 2005; pp 151191.

(26) Frauenrath, H. Prog. Polym. Sci. 2005, 30, 325-384.

(27) Percec, V.; Rudick, J. G.; Wagner, M.; Obata, M.; Mitchell, C. M.; Cho, W. D.; Magonov, S. N. Macromolecules 2006, 39, 7342-7351.

(28) Canilho, N.; Kasëmi, E.; Mezzenga, R.; Schlüter, A. D. J. Am. Chem. Soc. 2006, 128, 13998-13999.

(29) Canilho, N.; Kasëmi, E.; Schlüter, A. D.; Mezzenga, R. Macromolecules 2007, 40, 2822-2830.

(30) Kurth, D. G.; Lehmann, P.; Schutte, M. Proc. Natl. Acad. Sci. U.S.A. 2000, 97, 5704-5707

(31) Kurth, D. G.; Severin, N.; Rabe, J. P. Angew. Chem., Int. Ed. 2002, $41,3681-3683$

(32) Barbera, J.; Donnio, B.; Gehringer, L.; Guillon, D.; Marcos, M Omenat, A.; Serrano, J. L. J. Mater. Chem. 2005, 15, 4093-4105.

(33) Ruokolainen, J.; ten Brinke, G.; Ikkala, O. Adv. Mater. 1999, 11, 777780.

(34) Morkved, T. L.; Lu, M.; Urbas, A. M.; Ehrichs, E. E.; Jaeger, H. M.; Mansky, P.; Russell, T. P. Science 1996, 273, 931-933.

(35) Zhang, A.; Zhang, B.; Wächtersbach, E.; Schmidt, M.; Schlüter, A. D. Chem.-Eur. J. 2003, 9, 6083-6092.

(36) Zhang, A.; Okrasa, L.; Pakula, T.; Schlüter, A. D. J. Am. Chem. Soc. 2004, 126, 6658-6666.

(37) Kasëmi, E.; Zhuang, W.; Rabe, J. P.; Fischer, K.; Schmidt, M.; Colussi, M.; Keul, H.; Yi, D.; Colfen, H.; Schlüter, A. D. J. Am. Chem. Soc. 2006, 128, 5091-5099.

(38) Handlin, D. L.; Thomas, E. L. Macromolecules 1983, 16, 1514-1525.

(39) Williams, D. B.; Carter, C. B. Transmission Electron Microscopy: A Textbook for Materials Science; Springer: New York, 2004

(40) Donnio, B.; Heinrich, B.; Allouchi, H.; Kain, J.; Diele, S.; Guillon, D.; Bruce, D. W. J. Am. Chem. Soc. 2004, 126, 15258-15268.

(41) Mao, G.; Wang, J.; Clingman, S. R.; Ober, C. K.; Chen, J. T.; Thomas, E. L. Macromolecules 1997, 30, 2556-2567. 